\title{
Modeling of dielectric properties of complex fluids with an equation of state
}

\author{
Maribo-Mogensen, Bjørn; Kontogeorgis, Georgios M.; Thomsen, Kaj
}

Published in:

Journal of Physical Chemistry Part B: Condensed Matter, Materials, Surfaces, Interfaces \& Biophysical

Link to article, DOI:

10.1021/jp310572q

Publication date:

2013

Document Version

Publisher's PDF, also known as Version of record

Link back to DTU Orbit

Citation (APA):

Maribo-Mogensen, B., Kontogeorgis, G. M., \& Thomsen, K. (2013). Modeling of dielectric properties of complex fluids with an equation of state. Journal of Physical Chemistry Part B: Condensed Matter, Materials, Surfaces, Interfaces \& Biophysical, 117(12), 3389-3397. https://doi.org/10.1021/jp310572q

\section{General rights}

Copyright and moral rights for the publications made accessible in the public portal are retained by the authors and/or other copyright owners and it is a condition of accessing publications that users recognise and abide by the legal requirements associated with these rights.

- Users may download and print one copy of any publication from the public portal for the purpose of private study or research.

- You may not further distribute the material or use it for any profit-making activity or commercial gain

- You may freely distribute the URL identifying the publication in the public portal 


\title{
Modeling of Dielectric Properties of Complex Fluids with an Equation of State
}

\author{
Bjørn Maribo-Mogensen,* Georgios M. Kontogeorgis, and Kaj Thomsen
}

Center for Energy Resources Engineering, Department of Chemical and Biochemical Engineering, Technical University of Denmark (DTU), Søltofts Plads, Building 229, 2800 Kgs. Lyngby, Denmark

\section{Supporting Information}

ABSTRACT: The static permittivity is a key property for describing solutions containing polar and hydrogen bonding compounds. However, the precise relationship between the molecular and dielectric properties is not well-established. Here we show that the relative permittivity at zero frequency (static permittivity) can be modeled simultaneously with thermodynamic properties. The static permittivity is calculated from an extension of the framework developed by Onsager, Kirkwood, and Fröhlich to associating mixtures. The

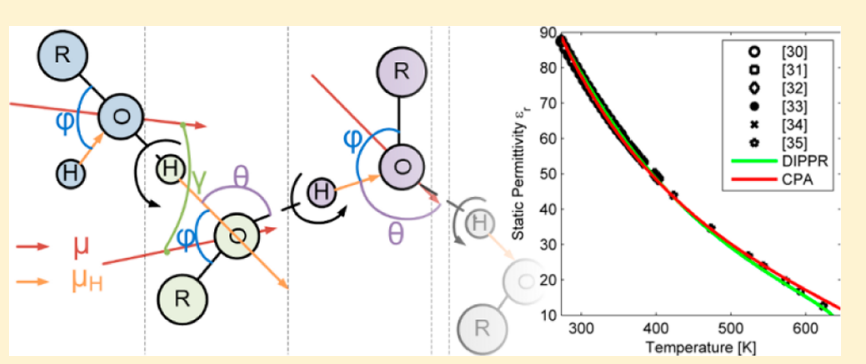
thermodynamic properties are calculated from the cubic-plusassociation (CPA) equation of state that includes the Wertheim association model as formulated in the statistical associating fluid theory (SAFT) to account for hydrogen bonding molecules. We show that, by using a simple description of the geometry of the association, we may calculate the Kirkwood $g$-factor as a function of the probability of hydrogen bond formation. The results show that it is possible to predict the static permittivity of complex mixtures over wide temperature and pressure ranges from simple extensions of well-established theories simultaneously with the calculation of thermodynamic properties.

\section{INTRODUCTION}

Dielectric spectroscopy is widely used to gain insight into molecular properties of fluids. ${ }^{1-4}$ The dielectric properties of fluids are characterized from the complex permittivity $\varepsilon^{\prime}+i \varepsilon^{\prime \prime}$, which can be measured from the response of the fluid to an external electrical field. ${ }^{1-4}$ The electrical response ultimately depends on many factors, including transport and thermodynamic properties (density, viscosity, etc.), molecular properties (dipole moment, molecular polarizability, etc.), and also the fluid structure (e.g., due to hydrogen bonding). ${ }^{1-9}$ The zerofrequency limit of the relative permittivity of a material, also known as the static relative permittivity, or the dielectric constant, is a measure of the ratio of the capacitance of a medium relative to the capacitance of a vacuum. ${ }^{1,2,9}$ The static permittivity is required as input to models for the Coulombic interactions between ions in a polar medium, ${ }^{10}$ such as the Debye-Hückel ${ }^{11}$ theory and the Born ${ }^{12}$ model of the solvation free energy.

The static permittivity has also been used to correlate solubility and speciation of neutral compounds and pharmaceuticals $^{13}$ and to predict the scaling propensity of produced water containing gas hydrate inhibitors. ${ }^{14}$ Online measurements of the permittivity are used for nondestructive sensing of moisture content of soils and food. ${ }^{4,15}$ It also serves as a valuable resource for assessment of water saturation in geological formations and determination of the hydrocarbon content in the presence of fresh formation water or water with unknown salinity. ${ }^{16}$
The theoretical background for predicting the static permittivity $\left(\varepsilon_{\mathrm{r}}\right)$ of polar compounds from molecular properties relies on the famous papers by Onsager ${ }^{5}$ and Kirkwood. ${ }^{6}$ Fröhlich ${ }^{7}$ introduced the Kirkwood $g$-factor accounting for the local structure in Onsager's relations, and Hasted ${ }^{4}$ extended the formulation to mixtures, as shown in eq 1 .

$$
\frac{\left(2 \varepsilon_{\mathrm{r}}+\varepsilon_{\infty}\right)\left(\varepsilon_{\mathrm{r}}-\varepsilon_{\infty}\right)}{\varepsilon_{\mathrm{r}}\left(\varepsilon_{\infty}+2\right)^{2}}=\frac{N_{\mathrm{A}}}{9 \varepsilon_{0} k_{\mathrm{B}} T v} \sum_{i} x_{i} g_{i} \mu_{i, 0}{ }^{2}
$$

In eq $1, \varepsilon_{\mathrm{r}}$ is the static permittivity, $\varepsilon_{\infty}$ is the permittivity at infinite frequency, $\varepsilon_{0}$ is the vacuum permittivity, $N_{\mathrm{A}}$ is Avogadro's constant, $T$ is the temperature, $k_{\mathrm{B}}$ is the Boltzmann constant, $x_{i}$ is the mole fraction of component $i, v$ is the molar volume, and $\mu_{i, 0}$ is the vacuum dipole moment of component $i$.

Equation 1 can be derived using the procedure by Buckingham ${ }^{17}$ and by assuming the same infinite frequency permittivity $\varepsilon_{\infty}$ for all spherical enclosures (see the full derivation in the Supporting Information). $\varepsilon_{\infty}$ is calculated from the Clausius-Mossotti ${ }^{1,2}$ equation for mixtures shown in eq 2 :

$$
\frac{\varepsilon_{\infty}-1}{\varepsilon_{\infty}+2}=\frac{1}{3 \varepsilon_{0}} \frac{N_{\mathrm{A}}}{v} \sum_{i} x_{i} \alpha_{0, i}
$$

Received: October 25, 2012

Revised: February 26, 2013

Published: March 4, 2013 
In eq 2, $\alpha_{0, i}$ is the molecular polarizability of molecule $i$. Oster and Kirkwood ${ }^{8}$ derived a model for the pure component $g$ factor for water and alcohols using eq 3 :

$$
g=1+z\langle\cos \gamma\rangle
$$

in which $z$ is the coordination number (four in the case of water) and $\langle\cos \gamma\rangle$ is a statistical mechanical average projected angle for the dipole moment of the surrounding molecules. Haggis et al. ${ }^{3}$ used the "broken-down-ice" structure of water, to accurately predict the static permittivity of water over wider temperature ranges by accounting for a reduction in the degree of hydrogen bonding. Suresh and Naik ${ }^{18,19}$ presented a model for predicting the dielectric constant of binary mixtures of methanol, water, and acetone by calculating $\langle\cos \gamma\rangle$ from molecular geometry and the probability of association determined by a chemical model for hydrogen bonding. Suresh improved the model for mixtures ${ }^{20}$ and used the model to analyze two proposed schemes for the cross-association between water and dimethyl sulfoxide.

This article presents a novel method for predicting the static permittivity of hydrogen-bonding mixtures over wide ranges of temperature and pressure using eq 1 through a geometrical model for calculation of Kirkwood's $g$-factor coupled with Wertheim's association theory within the cubic-plus-association (CPA) equation of state. ${ }^{21-23}$

Theoretical Basis. The following section summarizes the development of a new model for the static permittivity of mixtures containing hydrogen-bonding compounds. It extends the model for mixtures shown in eq 1 , in which the $g$-factor can be calculated from eq 4 (see derivation in the Supporting Information):

$$
g_{i}=1+\frac{1}{\mu_{0, i}^{2}}\left\langle\sum_{j \neq i} \boldsymbol{\mu}_{0, i} \cdot \boldsymbol{\mu}_{0, j}^{\prime}\right\rangle
$$

In eq $4, \boldsymbol{\mu}_{0, j}^{\prime}$ denotes the dipole moment of molecules surrounding the central dipole. We will approximate eq 4 from the projected dipole moments of all molecules that are hydrogen bonded to a fixed central molecule C. A sketch of the innermost neighbor is shown in Figure 1.

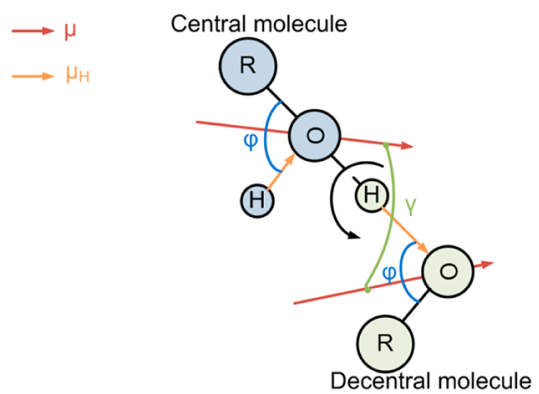

Figure 1. Sketch of bonding angles and dipole moments with the innermost neighbor.

We may calculate the angle between the two dipole moments $\gamma$ from the projection of the dipole moment of a decentral molecule $\boldsymbol{\mu}_{\mathrm{D}}$ onto the central molecule $\boldsymbol{\mu}_{\mathrm{C}}$ using eq 5 :

$$
\langle\cos \gamma\rangle=\frac{\left\langle\boldsymbol{\mu}_{\mathrm{C}} \cdot \boldsymbol{\mu}_{\mathrm{D}}\right\rangle}{\mu_{\mathrm{C}} \mu_{\mathrm{D}}}
$$

If we assume free rotation around the hydrogen bond, we may write eq 5 in terms of the dipole moment in the direction of the hydrogen bond $\boldsymbol{\mu}_{\mathrm{H}}$ toward the other molecule:

$$
\langle\cos \gamma\rangle=\frac{\boldsymbol{\mu}_{\mathrm{H}} \cdot \boldsymbol{\mu}_{\mathrm{D}}}{\mu_{\mathrm{C}} \mu_{\mathrm{D}}}
$$

Using simple trigonometric relationships to evaluate eq 6, we may obtain eq 7 for tetrahedral and eq 8 for planar networks (we refer to the derivation shown in the Supporting Information):

$$
\begin{aligned}
\langle\cos \gamma\rangle= & -\frac{\mu_{\mathrm{OH}}+\mu_{\mathrm{OR}} \cos \varphi}{\mu_{\mathrm{C}} \mu_{\mathrm{D}}}\left(\mu_{\mathrm{OH}} \cos \theta+\mu_{\mathrm{OR}}\right. \\
& \left.\left(\cos \theta \cos \varphi-\frac{1}{2} \sin \theta \sin \varphi\right)\right) \\
\langle\cos \gamma\rangle= & -\frac{\mu_{\mathrm{OH}}+\mu_{\mathrm{OR}} \cos \varphi}{\mu_{\mathrm{C}} \mu_{\mathrm{D}}}\left(\mu_{\mathrm{OH}} \cos \theta+\mu_{\mathrm{OR}}\right. \\
& (\cos \theta \cos \varphi-\sin \theta \sin \varphi))
\end{aligned}
$$

In eqs 7 and $8, \mu_{\mathrm{OH}}$ and $\mu_{\mathrm{OR}}$ are the constituents to the dipole moment in the $\mathrm{O}-\mathrm{H}$ and $\mathrm{O}-\mathrm{R}$ directions, respectively. Equations 7 and 8 may be used for pure components and mixtures to determine angles between proton donors and proton acceptors and vice versa. For predictions, we use $\theta=$ $109.47^{\circ}$ for the tetrahedral network and $\theta=120^{\circ}$ for the planar network.

Assuming that the central molecule can form up to $z$ hydrogen bonds, we may calculate the contribution to the scalar product of the dipole moments of the first $z$ neighbors using eq 9:

$$
\left\langle\boldsymbol{\mu}_{\mathrm{C}} \cdot \boldsymbol{\mu}_{\mathrm{D}}\right\rangle^{(1)}=z \mu_{\mathrm{C}} \mu_{\mathrm{D}}\langle\cos \gamma\rangle
$$

In order to determine the projected dipole moment from all neighbors to the central molecule, we construct a simplified picture of the hydrogen bonding network, as shown in Figure 2, that includes hydrogen-bonded molecules from the first, second, and $n$th shell.

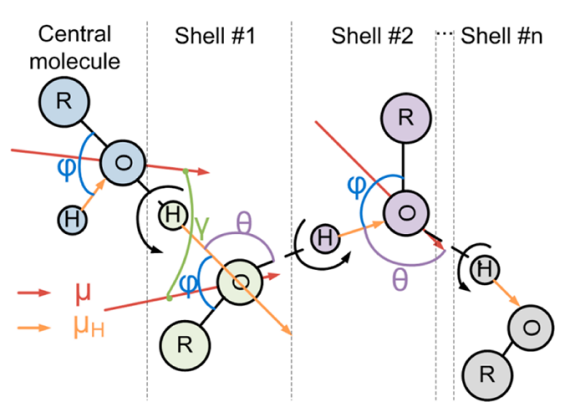

Figure 2. Sketch of color-coded important dipole moments and angles in a hydrogen-bonding system.

If the molecules can form more than two hydrogen bonds, each shell will contain more molecules than the first. As shown by Suresh and Naik, ${ }^{18}$ the geometrical configurations in the tetrahedrally coordinated shells result in the dipole moments of all but $z$ molecules being canceled out. We will therefore assume that there are only $z$ molecules in each shell that give a net contribution to the central dipole moment. The projected dipole moment from the $k$ th shell onto the first shell may then 
be calculated from the rotation angle $\theta$ which is multiplied onto the projected dipole moment of the first shell using eq 10 :

$$
\left\langle\boldsymbol{\mu}_{\mathrm{C}} \cdot \boldsymbol{\mu}_{\mathrm{D}}\right\rangle^{(k)}=\left\langle\boldsymbol{\mu}_{\mathrm{C}} \cdot \boldsymbol{\mu}_{\mathrm{D}}\right\rangle^{(1)} \cos ^{k-1}(\pi-\theta), \quad k=2 \ldots \infty
$$

We will then approximate the statistical mechanical average in eq 4 for mixtures of associating compounds by a summation over the surrounding shells for all molecules given by eq 11 :

$$
\left\langle\sum_{j \neq i} \boldsymbol{\mu}_{0, i} \cdot \boldsymbol{\mu}_{0, j}^{\prime}\right\rangle=\left\langle\sum_{k}^{\text {shells }} \sum_{j}^{\text {components }}\left\langle\boldsymbol{\mu}_{0, i} \cdot \boldsymbol{\mu}_{0, j}^{\prime}\right\rangle\right\rangle
$$

The statistical mechanical average $\left\langle\boldsymbol{\mu}_{0, i} \boldsymbol{\mu}_{0, j}^{\prime}\right\rangle$ depends on the probability of molecules $i$ and $j$ being associated $\left(P_{i j}\right)$ and on the projection of the dipole moment onto the central molecule.

Using eq 9, we may calculate the statistical mechanical average of the projection of the dipole moments from the first hydration shell onto the central molecule using eq 12, in which we have dropped the bracket notation \langle\rangle for the statistical mechanical average angle:

$$
\sum_{j}\left\langle\boldsymbol{\mu}_{0, i} \cdot \boldsymbol{\mu}_{0, j}^{\prime}\right\rangle=\mu_{0, i} \sum_{j} z_{i j} P_{i j} \mu_{0, j} \cos \gamma_{i j}
$$

In eq $12, z_{i j}$ is the coordination number of molecule $j$ around a central molecule $i$ and the angle $\cos \gamma_{i j}$ corresponds to the angle between the two dipole moments, as shown in Figure 1. The contributions from the second, third, and the $k$ th shells are calculated using eq 10 . The probability of the $k$ th shell to exist is equal to $P_{i}^{k}$, where $P_{i}=\sum_{j \in S} P_{i j}$ is the probability of the molecule $i$ to be bonded to any of the molecules in the set $S$. The contribution from the $k$ th shell to eq 11 is then given by eq 13:

$$
\sum_{j}\left\langle\mu_{0, i} \cdot \mu_{0, j}^{\prime}\right\rangle^{(k)}=\boldsymbol{\mu}_{0, i} \sum_{j} z_{i j} P_{i j} \boldsymbol{\mu}_{0, j} \cos \gamma_{i j}\left[P_{i} \cos \left(\pi-\theta_{i j}\right)\right]^{k}
$$

We may then calculate the sum of the contributions over all shells, as shown in eq 14 :

$$
\left\langle\sum_{k}^{\text {shells }} \sum_{j}^{\text {components }}\left\langle\boldsymbol{\mu}_{0, i} \cdot \boldsymbol{\mu}_{0, j}^{\prime}\right\rangle\right\rangle=\mu_{0, i} \sum_{j} \frac{z_{i j} P_{i j} \mu_{0, j} \cos \gamma_{i j}}{P_{i} \cos \theta_{i j}+1}
$$

By inserting eq 14 in eq 4, we obtain the explicit expression shown in eq 15 for the $g$-factor in mixtures of associating compounds.

$$
g_{i}=1+\sum_{j} \frac{z_{i j} P_{i j} \cos \gamma_{i j}}{P_{i} \cos \theta_{i j}+1} \frac{\mu_{0, j}}{\mu_{0, i}}
$$

Note that eq 15 does not account for closed (e.g., ring-like) structures. Furthermore, the parameters for eq 15 cannot easily be deduced for components with multiple conformers and must be fitted to experimental data. Note also that if $\cos \gamma_{i j}$ is negative (corresponding to the dipoles being aligned antiparallelly), eq 15 may result in $g<1$.

Examples. By applying eq 7 in the case of water $\left(\mu_{0}=1.855\right.$ $\mathrm{D}, \varphi=104.5^{\circ}, \theta=109.5^{\circ}$ ), we obtain $\gamma=69.4^{\circ}$. By assuming that the bond length of the $\mathrm{O}-\mathrm{H}$ bond does not change significantly in water compared to alcohols and by using the value $\mu_{\mathrm{OH}}=1.52 \mathrm{D}$ from water for methanol, we calculate the angle for methanol $\left(\mu_{0}=1.7 \mathrm{D}\right.$ yields the angle $\gamma=57.8^{\circ}$ with a planar configuration).
The nature of the problem changes in the case of multifunctional molecules and molecules with internal degrees of freedom, as these conformational changes may affect the overall magnitude and direction of the dipole moment. In such situations, the total mixture may contain different conformers of the molecules and essentially requires us to perform a calculation of the distribution of conformers and then perform calculations for each geometrical configuration. In general, we have to fit the parameters $\cos \gamma$ and $\cos \theta$ to experimental data for such cases, but if the geometrical configuration and coordination number are known, we may fit the data using $\cos \theta$ and eq 7 or eq 8 .

Selection of Hydrogen Bonding Network. Before any calculations of the static permittivity can be performed, it is necessary to determine the hydrogen bonding network. Using experimental data for the static permittivity, we may determine the Kirkwood $g$-factor by rearranging eq 1 to form eq 16 :

$$
g_{i}=\frac{1}{\mu_{i}^{2}} \frac{\left(2 \varepsilon_{\mathrm{r}}+\varepsilon_{\infty}\right)\left(\varepsilon_{\mathrm{r}}-\varepsilon_{\infty}\right)}{\varepsilon_{\mathrm{r}}\left(\varepsilon_{\infty}+2\right)^{2}} \frac{9 \varepsilon_{0} k_{\mathrm{B}} T v}{N_{\mathrm{A}}}
$$

The $g$-factor calculated near the freezing point temperature (i.e., at a high degree of association) serves as a good indicator for the choice of the hydrogen bonding network. It may be compared to the maximum $g$-factor that is calculated from the model by inserting $P_{i j}=1$ into eq 15 for a pure component:

$$
g_{\max }=1+\frac{z_{i j} \cos \gamma_{i j}}{\cos \theta_{i j}+1}
$$

To illustrate how the choice of hydrogen network affects the calculated $g$-factors, we calculate the experimental $g$-factor for methanol in the range $3.25-3.5$ at $163-183 \mathrm{~K}$. If methanol was in a tetrahedral configuration $\left(\theta=109.47^{\circ}\right)$ with a maximum of three hydrogen bonds (from the two lone pairs on oxygen and the one hydrogen bond), the maximum $g$-factor calculated using eqs 17 and 7 is 2.76, and thus, this hydrogen bonding configuration cannot reproduce the experimental data. If we instead use a planar configuration $\left(\theta=120^{\circ}\right)$ with two hydrogen bonds, the maximum $g$-factor calculated using eqs 17 and 8 is 3.67 , which is in much better accordance with the observed values.

Thermodynamic Model. The $\mathrm{CPA}^{21-23}$ equation of state (EoS) consists of the Soave-Redlich-Kwong (SRK) ${ }^{24}$ cubic EoS with the Wertheim association theory, ${ }^{25}$ as formulated by the statistically associated fluid theory $(\mathrm{SAFT})^{26}$ to account for association of hydrogen bonding components. The model has been used to calculate phase equilibrium and liquid densities for many complex mixtures, especially in relation to the phase equilibrium of water, oil, natural gas, and chemicals. The pressure equation of CPA is calculated from eq $18^{21-23}$

$$
\begin{aligned}
P= & \frac{R T}{v-b}-\frac{a(T)}{v(v+b)}-\frac{1}{2} \frac{R T}{v}\left(1+\rho \frac{\partial \ln g}{\partial \rho}\right) \\
& \sum_{i} x_{i} \sum_{A_{i}}\left(1-X_{A_{i}}\right)
\end{aligned}
$$

In eq $18, P$ is the pressure, $R$ is the ideal gas constant, $T$ is the temperature, $b$ is the covolume parameter of the mixture, $x_{i}$ is the mole fraction of molecule $i, v$ is the molar volume, $\rho$ is the molar density, $X_{A_{i}}$ is the fraction of molecules of component $i$ not bonded at site $A$, and the radial distribution function in CPA is calculated from $g(\rho)=(1-1.9 \eta)^{-1}$, where the packing 
Table 1. CPA Parameters, Association Volumes, and Reduced Energy for Selected Components ${ }^{21-23}$

\begin{tabular}{|c|c|c|c|c|c|c|c|}
\hline component & $T_{\mathrm{c}}(\mathrm{K})$ & $b\left(\mathrm{~cm}^{3} / \mathrm{mol}\right)$ & $\Gamma=\left(a_{0} / R b\right)(\mathrm{K})$ & $c_{1}$ & $\nu_{A_{i j} B_{j}}\left(\mathrm{~cm}^{3} / \mathrm{mol}\right)$ & $\varepsilon_{A_{i j} B_{j}} / k_{\mathrm{B}}(\mathrm{K})$ & association scheme \\
\hline water & 647.13 & 14.52 & 1017.3 & 0.6736 & 1.004 & 2003.25 & $4 \mathrm{C}$ \\
\hline methanol & 512.64 & 30.98 & 1573.7 & 0.4310 & 0.499 & 2957.78 & $2 \mathrm{~B}$ \\
\hline ethanol & 513.92 & 49.11 & 2123.8 & 0.7369 & 0.393 & 2589.85 & $2 \mathrm{~B}$ \\
\hline propan-1-ol & 536.78 & 64.11 & 2234.5 & 0.9171 & 0.519 & 2525.86 & $2 \mathrm{~B}$ \\
\hline butan-1-ol & 563.05 & 79.70 & 2368.6 & 0.9784 & 0.654 & 2525.86 & $2 \mathrm{~B}$ \\
\hline octan-1-ol & 652.5 & 148.5 & 3368.0 & 1.1486 & 0.0208 & 3218.55 & $2 \mathrm{~B}$ \\
\hline ethylene glycol & 719.7 & 51.40 & 2531.7 & 0.6744 & 0.725 & 2375.75 & $4 \mathrm{C}$ \\
\hline diethylether & 466.7 & 92.36 & 2302.7 & 0.5946 & & & \\
\hline hydrogen sulfide & 373.53 & 28.5 & 1878.2 & 0.6027 & & & \\
\hline hexane & 507.6 & 107.9 & 2640.0 & 0.8313 & & & \\
\hline decane & 617.7 & 178.7 & 3190.5 & 1.1324 & & & \\
\hline
\end{tabular}

Table 2. Pure Component Properties for Calculating the Static Permittivity Using Densities from DIPPR Correlations and the Deviation from Experimental Data

\begin{tabular}{|c|c|c|c|c|c|c|c|c|c|c|}
\hline \multirow[b]{2}{*}{ component } & \multirow[b]{2}{*}{$\alpha_{0} \times 10^{40}\left[\left(\mathrm{C}^{2} \mathrm{~m}^{2}\right) / \mathrm{J}\right]$} & \multirow[b]{2}{*}{$\mu_{0}(\mathrm{D})$} & \multicolumn{2}{|c|}{$\varepsilon_{\mathrm{r}}$ at $20^{\circ}$} & \multirow[b]{2}{*}{$\gamma(\mathrm{deg})$} & \multirow[b]{2}{*}{$\varphi(\operatorname{deg})$} & \multirow[b]{2}{*}{$\theta(\operatorname{deg})$} & \multirow[b]{2}{*}{$z(\operatorname{deg})$} & \multirow[b]{2}{*}{$\mathrm{AAD}$} & \multirow[b]{2}{*}{$\mathrm{RAD}(\%)$} \\
\hline & & & Calc. & Exp. & & & & & & \\
\hline \multicolumn{11}{|c|}{ Associating Components } \\
\hline water & 1.613 & 1.855 & 78.8 & 80.1 & 69.5 & 104.5 & $108.9^{*}$ & $4(\mathrm{~T})$ & 0.80 & 3.04 \\
\hline methanol & 3.661 & 1.70 & 34.0 & 33.0 & 52.7 & 109.47 & $118.7^{*}$ & $2(\mathrm{P})$ & 0.8 & 1.9 \\
\hline ethanol & 6.019 & 1.69 & 25.2 & 25.3 & 52.3 & 109.47 & $120.1^{*}$ & $2(\mathrm{P})$ & 1.0 & 4.9 \\
\hline propanol & 7.499 & 1.68 & 20.8 & 20.8 & 51.8 & 109.47 & $124.5^{*}$ & $2(\mathrm{P})$ & 0.6 & 2.6 \\
\hline 1-butanol & 9.880 & 1.66 & 18.5 & 17.8 & 50.8 & 109.47 & $126.8^{*}$ & $2(\mathrm{P})$ & 0.3 & 2.6 \\
\hline 1-pentanol & 11.80 & 1.70 & 15.4 & 15.1 & 52.7 & 109.47 & $127.8 *$ & $2(\mathrm{P})$ & 0.4 & 3.1 \\
\hline 1-hexanol & 13.84 & 1.65 & 12.8 & 13.0 & 50.3 & 109.47 & $127.9 *$ & $2(\mathrm{P})$ & 0.3 & 2.8 \\
\hline 1-octanol & 17.92 & 1.76 & 10.6 & 10.3 & 55.4 & 109.47 & $126.7^{*}$ & $2(\mathrm{P})$ & 0.2 & 2.0 \\
\hline ethylene glycol & 6.342 & 2.36 & 43.4 & 41.4 & 72.4 & 109.47 & $115.6^{*}$ & $3(\mathrm{P})$ & 0.8 & 2.0 \\
\hline \multicolumn{11}{|c|}{ Nonassociating } \\
\hline \multirow[t]{2}{*}{ diethylether } & 11.35 & 1.3 & 4.39 & 4.27 & & & & & 0.3 & 4.7 \\
\hline & & $1.43^{*}$ & & & & & & & 0.1 & 2.0 \\
\hline hydrogen sulfide & 4.395 & 0.978 & 5.17 & 5.93 & & & & & 0.6 & 12.8 \\
\hline hexane & 13.24 & & 1.89 & 1.89 & & & & & 0.00 & 0.4 \\
\hline decane & 21.25 & & 1.99 & 1.99 & & & & & 0.01 & 0.3 \\
\hline
\end{tabular}

factor $\eta$ is given by $b / 4 v$. For mixtures, CPA uses the vdW1f mixing rules for the SRK $a(T)$ and $b$ parameters, as shown in eqs 19 and 20:

$$
\begin{aligned}
& a(T)=\sum_{i} \sum_{j} x_{i} x_{j} \sqrt{a_{i}(T) a_{j}(T)}\left(1-k_{i j}\right) \\
& b=\sum_{i} x_{i} b_{i}
\end{aligned}
$$

in which $a_{i}(T)$ is given by eq 21 :

$$
a_{i}(T)=a_{0 i}\left(1+c_{1 i}\left(1-\sqrt{T / T_{c, i}}\right)\right)^{2}
$$

In eq $21, a_{0 i}$ and $c_{1 i}$ are pure component parameters and $T_{c, i}$ is the critical temperature of component $i$. Given a pressure and a temperature, eq 18 is solved for the volume root $v$ of the desired phase. Phase equilibrium is determined from the fugacity coefficients calculated using the EoS.

The CPA EoS uses the covolume parameter $b, \Gamma_{i}=a_{0 i} / R b_{i}$ and $c_{1 i}$ as the pure component parameters for the physical part (SRK), and the association energy $\varepsilon_{A B}$ and association volume $\nu$ as the parameters for the association model. The CPA parameters were estimated by fitting to experimental vapor pressure and liquid densities. ${ }^{22,23} \mathrm{We}$ adopt the model parameters fitted to thermodynamic properties, as shown in Table 1.
In connection with prediction of the static permittivity, CPA is used to calculate the volume and the probability of association, which is already calculated by solving eq 22 within the equation of state:

$$
\frac{1}{X_{A_{i}}}=1+\frac{1}{v} \sum_{j} x_{j} \sum_{B_{j}} X_{B_{j}} \Delta_{A_{i} B_{j}}
$$

In eq 22, $X_{A_{i}}$ is the fraction of site $A$ on molecule $i$ that is not bonded to any other component and $\Delta_{A_{i} B_{j}}$ is the association strength (equilibrium constant), which in CPA is calculated from eq 23:

$$
\Delta_{A_{i} B_{j}}=g(\rho) v_{A_{i} B_{j}}\left[\exp \left(\frac{\varepsilon_{A_{i} B_{j}}}{k_{\mathrm{B}} T}\right)-1\right]
$$

In eq $23, v_{A_{i} B_{j}}$ is the association volume and $\varepsilon_{A_{j} B_{j}}$ the association energy. The probability of two sites to be hydrogen bonded is calculated from eq 24 , where $X_{A_{i}}$ denotes the fraction of sites of type $A$ on molecule $i$ that are not associated to other sites.

$$
P_{A_{i} B_{j}}=\rho x_{j} \Delta_{A_{i} B_{j}} X_{A_{i}} X_{B_{j}}
$$

For a pure component, the probability of two molecules to be bonded may be obtained using eq 25 : 


$$
P_{i j}=\sum_{B_{j}} P_{A_{i} B_{j}}=1-X_{A_{i}}
$$
15.

We use eq 24 to determine the Kirkwood $g$-factor from eq

\section{RESULTS AND DISCUSSION}

The static permittivity has been calculated for pure compounds using the densities calculated from correlations available in the

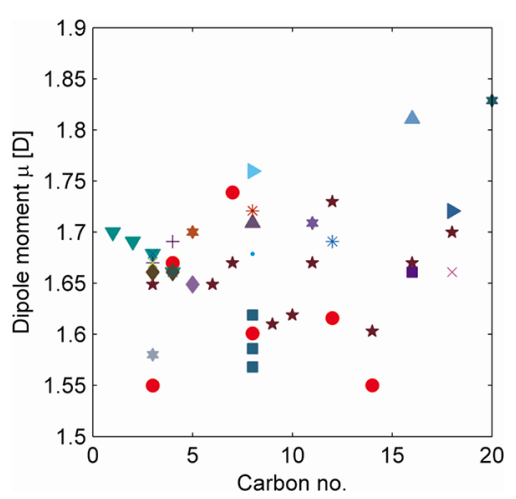

Figure 3. Scatter of reported dipole moments for alcohols available in the DIPPR 801 database. ${ }^{28}$ Symbols indicate different sources.

Table 3. Refitted Pure Component Properties for Calculation of Static Permittivity Using Densities Calculated by CPA and Deviation from Experimental Data from the Landolt Börnstein Database ${ }^{29}$

\begin{tabular}{lcccccc} 
& \multicolumn{2}{c}{$\varepsilon_{\mathrm{r}}$ at $20^{\circ}$} & & & & \\
\cline { 2 - 4 } \multicolumn{1}{c}{ component } & calc. & exp. & $\gamma(\mathrm{deg})$ & $\theta(\mathrm{deg})$ & AAD & RAD (\%) \\
water & 80.2 & 80.1 & $63.5^{*}$ & $95.80^{*}$ & 0.69 & 3.98 \\
methanol & 34.3 & 33.0 & 52.9 & $118.6^{*}$ & 0.82 & 1.9 \\
ethanol & 24.8 & 25.3 & 52.1 & $122.1^{*}$ & 0.88 & 3.8 \\
propanol & 20.7 & 20.8 & 51.3 & $126.8^{*}$ & 0.45 & 1.7 \\
1-butanol & 18.4 & 17.8 & 50.13 & $128.6^{*}$ & 0.6 & 4.4 \\
1-pentanol & 14.9 & 15.1 & 52.4 & $130.4^{*}$ & 0.35 & 3.0 \\
1-hexanol & 12.9 & 13.0 & 49.6 & $128.0^{*}$ & 0.32 & 3.4 \\
1-octanol & 10.0 & 10.3 & 55.6 & $132.4^{*}$ & 0.19 & 2.2 \\
ethylene glycol & 41.6 & 41.4 & 72.63 & $119.62^{*}$ & 0.8 & 2.0
\end{tabular}

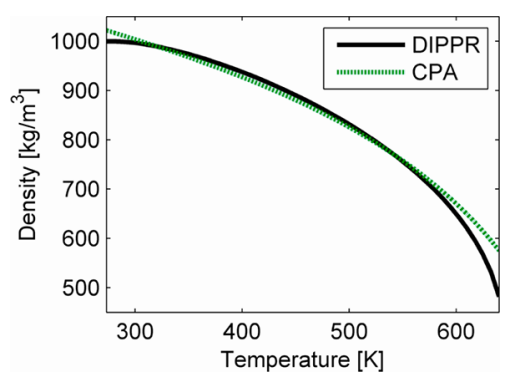

Figure 4. Comparison of the calculated liquid density of water using CPA and DIPPR. ${ }^{28}$

DIPPR database ${ }^{28}$ and the probability of association calculated from solving eq 22 using the parameters from Table 1 . The results are summarized in Table 2. While it is possible to obtain a good correspondence with experimental data (within $5 \%$ RAD for most fluids) by only fitting the parameter $\theta$ to $\varepsilon_{\mathrm{r}}$ at 20 ${ }^{\circ} \mathrm{C}$, the parameters shown in Table 2 were fine-tuned against all available data.

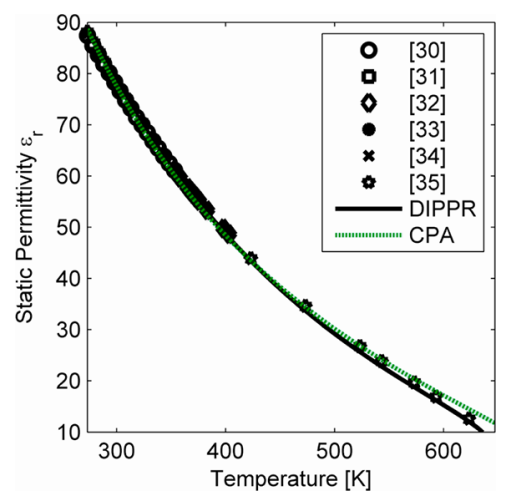

Figure 5. Comparison of experimental data ${ }^{30-35}$ for static permittivity of water and those calculated from the presented model using DIPPR and CPA densities.

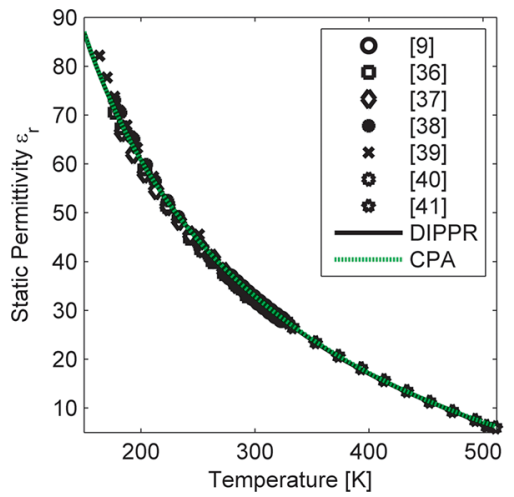

Figure 6. Comparison of experimental data ${ }^{9,36-41}$ for static permittivity of pure methanol as a function of temperature.

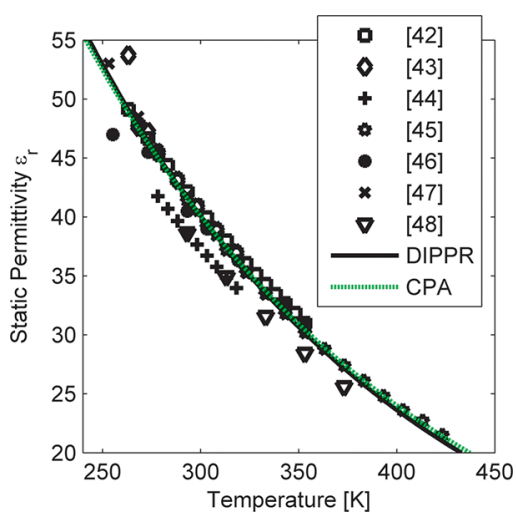

Figure 7. Comparison of experimental data ${ }^{42-48}$ for static permittivity of ethylene glycol as a function of temperature. Data from Kundu et al. $^{44}$ and Akerlöf ${ }^{48}$ were not used in the fit.

Table 4. Binary Parameters for Calculation of Static Permittivity of Mixtures of Water, Methanol, and Ethylene Glycol $^{21-23}$

\begin{tabular}{lcc}
\multicolumn{1}{c}{ binary mixture } & $\gamma_{i j}$ & $k_{i j}$ \\
water-methanol & $69.62^{\circ}$ & -0.09 \\
water-ethylene glycol & $69.72^{\circ}$ & -0.0115 \\
kmethanol-ethylene glycol & $66.35^{\circ}$ & 0.0 \\
\hline
\end{tabular}

Unless stated otherwise, the values for polarizability, dipole moment, and static permittivity are from the CRC Handbook of Chemistry and Physics ${ }^{27}$ and the DIPPR database. ${ }^{28}$ The 


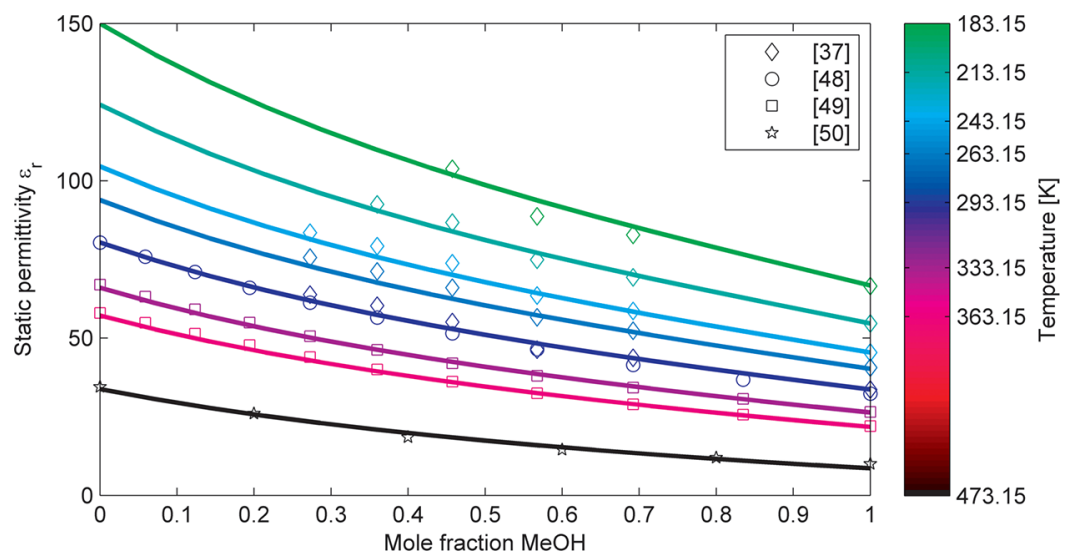

Figure 8. Static permittivity of water-methanol mixture at different temperatures. ${ }^{37,48-50}$ The data at $473.15 \mathrm{~K}$ from Teutenberg et al. ${ }^{50}$ is measured at $100 \mathrm{bar}$, the remaining at $1 \mathrm{bar}$ or the saturation pressure.

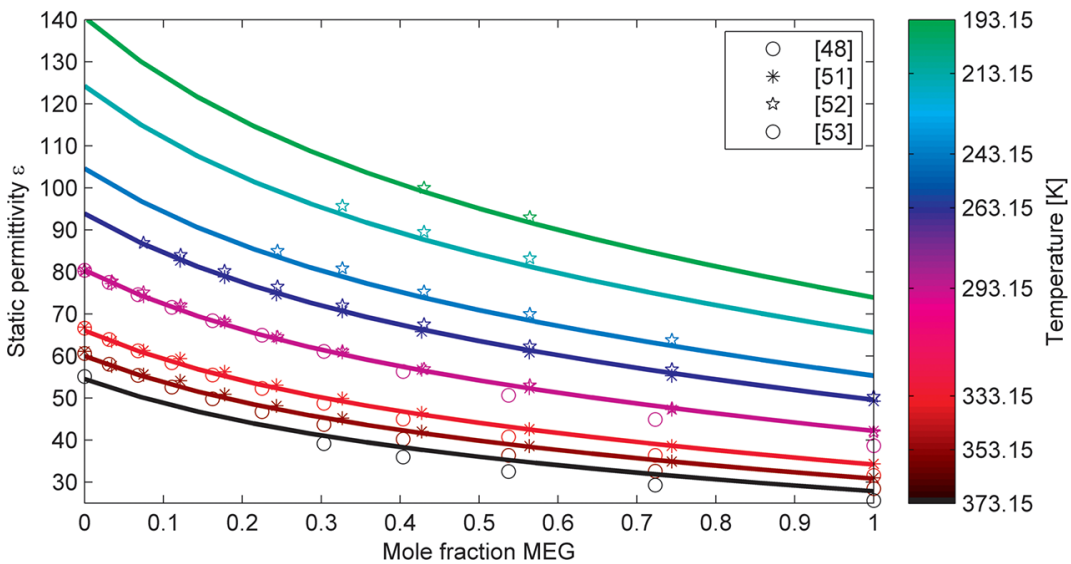

Figure 9. Static permittivity of water-ethylene glycol mixture. ${ }^{48,51-53}$ Data by Akerlö ${ }^{48}$ systematically display a lower static permittivity in ethylene glycol rich mixtures, which is attributed to experimental error.

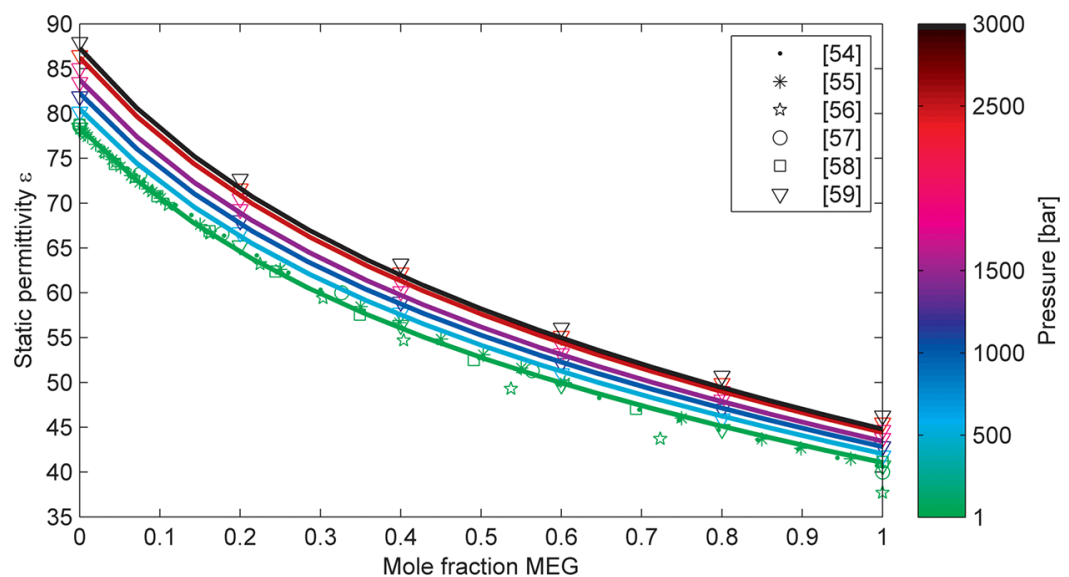

Figure 10. Static permittivity of mixtures of ethylene glycol and water at $25{ }^{\circ} \mathrm{C}$ as a function of pressure..$^{54-59}$

experimental data was from the Landolt-Börnstein database. ${ }^{29} *$ indicates a fitted value. $(\mathrm{T})$ indicates tetrahedral configuration (i.e., using eq 7), and (P) indicates planar (i.e., using eq 8).

Table 2 generally shows excellent agreement with the experimental data for static permittivity of the pure compounds that are hydrogen bonding. The biggest deviation is observed for hydrogen sulfide, which is not calculated as an associating component, and thus eq 1 reduces to the Onsager equation. For hexane and $\mathrm{n}$-decane, the static permittivity $\varepsilon_{\mathrm{r}}$ becomes equal to the infinite frequency permittivity $\varepsilon_{\infty}$. However, one of the difficulties arising for accurate calculation of the dielectric constant is the scatter in the experimental data reported in the literature for the vacuum dipole moment (see Figure 3 for the case of 1-alcohols as a function of chain length). In general, we use the dipole moment recommended by the DIPPR database, ${ }^{28}$ but in cases where this does not match the data well, we may have to fit the dipole moment along with $\cos \theta$.

The results indicate that the model is suitable for calculation of the static permittivity of pure components; however, in general, we are interested in the prediction of static permittivity 


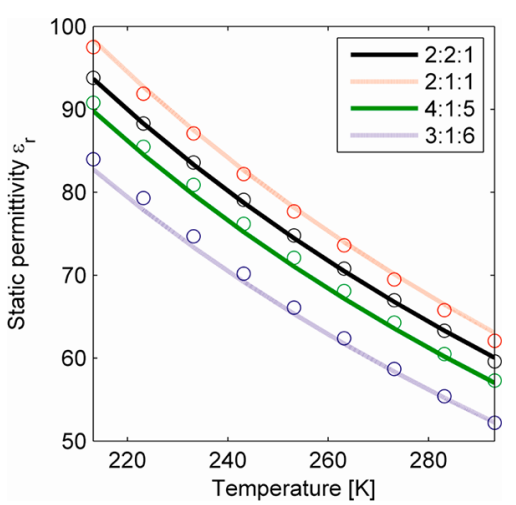

Figure 11. Static permittivity of mixtures of different volume fractions (prepared at $20^{\circ} \mathrm{C}$ ) of water-ethylene glycol-methanol, as a function of temperature. Data from Travers et al. ${ }^{60}$

for multicomponent systems over wide ranges of temperature and pressure. To enable calculation for multicomponent systems, we couple the model with the CPA EoS using the calculation procedure shown below:

- Specification of temperature, pressure, and composition

- Solve numerically for liquid volume (eq 18)

- Solve association equations numerically (eq 22)

- Calculate static permittivity (eqs 1 and 15)

While the CPA parameters are fitted to vapor pressure and liquid density, the CPA EoS does not always yield perfect agreement with DIPPR densities. This affects the calculation of the static permittivity in accordance with eq 1 , and it is therefore necessary to adjust the parameters slightly to match the experimental data. Table 3 shows the results and parameters when densities are calculated using CPA.

In the case of water, the unsatisfactory representation of the liquid density (see Figure 4) made it necessary to fit both angles $(\theta$ and $\gamma)$ to obtain a satisfactory fit with $\mathrm{RAD}<5 \%$. For the other compounds, it was only necessary to adjust $\theta$.

A comparison of the static permittivity of pure water calculated from DIPPR and CPA densities is shown in Figure 5.

A comparison of the present model with experimental data for methanol and ethylene glycol is presented in Figures 6 and 7 , showing good correspondence with the experimental data.

Mixtures. In the case of mixtures, we need to determine bonding angles, coordination numbers, and the type of hydrogen bonding network. While we may fit the parameters to experimental data, in this work, we predict the binary bonding angles from eq 7 assuming tetrahedral $\left(\theta=109.47^{\circ}\right)$ hydrogen bonding networks in the mixtures. We assume that methanol coordinates three molecules in its tetrahedral configuration, and that ethylene glycol and water will coordinate up to four molecules. The binary parameters are summarized in Table 4.

We use the near-Elliott combining rule ${ }^{21}$ shown in eqs 26 and 27 to determine cross-association energies and volumes between positive and negative sites on the molecules:

$$
\begin{aligned}
& v_{A_{i} B_{j}}=\sqrt{v_{A_{i} B_{i}}} \sqrt{v_{A_{j} B_{j}}} \\
& \varepsilon_{A_{i} B_{j}}=\frac{\varepsilon_{A_{i} B_{i}}+\varepsilon_{A_{j} B_{j}}}{2}
\end{aligned}
$$

Since no parameters are fitted to the experimental data, the results shown in this section are predictions from the pure component parameters.
Figure 8 compares the predicted static permittivity of mixtures of water and methanol with the experimental data as a function of temperature and composition. Figure 8 reveals good agreement with the experimental data, except for the sudden jump in static permittivity at low methanol mole fractions observed in the data from Travers and Douzou, ${ }^{37}$ which is attributed to experimental error.

Figure 9 shows results for the temperature dependence of the static permittivity in the binary water-ethylene glycol system. Note that the data from Akerlö ${ }^{48}$ are consistently underestimating the static permittivity of ethylene glycol rich mixtures. This deviation is attributed to experimental error, as the data for pure component ethylene glycol from Akerlöf ${ }^{48}$ shown in Figure 7 is significantly lower than data from other sources.

Figure 10 shows the static permittivity of water-ethylene glycol mixtures at $25{ }^{\circ} \mathrm{C}$ as a function of pressure and composition. Figure 10 shows that the new model captures the pressure dependence up to high pressures (2500 bar).

Figure 11 shows the predicted static permittivity with the experimental data of the ternary mixture containing water, methanol, and ethylene glycol as a function of temperature. Ethylene glycol is assumed to coordinate four water and methanol in a tetrahedral configuration.

As seen by Figures 8-11, the static permittivity of binary and ternary mixtures of associating compounds is well predicted by the present model. No new parameters were fitted to the binary or ternary systems; instead, the model parameters were calculated directly from eq 7 , and thus the presented results arise from true predictions of the model.

\section{CONCLUSION}

This work has extended the framework for calculating the static permittivity to associating compounds using an equation of state based on the Wertheim association theory. A model for the geometrical arrangements in hydrogen-bonding systems has been derived, and it was shown how this model allows for prediction of the static permittivity of pure compounds and mixtures over wide ranges of temperature and pressure when used within an equation of state using the association term by Wertheim. The model does not account for ring-type structures, or multiple conformers, but it is our experience that the model parameters can be tuned to match experimental data. Fitting one parameter to the static permittivity at $20{ }^{\circ} \mathrm{C}$ is sufficient for most compounds, provided that the density is accurately reproduced by the equation of state. The static permittivity of mixtures may be predicted solely from parameters calculated by a model of the geometrical configuration of the hydrogen-bonding structure involving the binary compounds.

\section{ASSOCIATED CONTENT}

\section{S Supporting Information}

Derivation of eqs 1,7 , and 8 . This material is available free of charge via the Internet at http://pubs.acs.org.

\section{AUTHOR INFORMATION}

\section{Corresponding Author}

*Address: Soltofts Plads, Building 229, Room 254, 2800 Kgs. Lyngby, Denmark. E-mail: bmm@kt.dtu.dk. Phone: +45 45252869. Fax: +4545882258 . 


\section{Notes}

The authors declare no competing financial interest.

\section{ACKNOWLEDGMENTS}

We thank the Technical University of Denmark and the CHIGP project joint industry consortium for funding this research.

\section{LIST OF SYMBOLS}

$b$ : SRK covolume

$g_{i}$ : Kirkwood $g$-factor for molecule $i$

$g(\rho)$ : radial distribution function

$k_{\mathrm{B}}$ : Boltzmann constant $\left(1.3806503 \times 10^{-23} \mathrm{~J} / \mathrm{K}\right)$

$N_{\mathrm{A}}$ : Avogadro number

$P$ : pressure

$P_{A_{i} B_{j}}$ : probability of association between site $A$ on molecule $i$ and site $B$ on molecule $j$

$P_{i j}$ : probability of molecule $i$ and $j$ to be hydrogen bonded

$P_{i}$ : probability of molecule $i$ to be bonded to any other molecules

$R$ : ideal gas constant $(8.3144621 \ldots \mathrm{J} / \mathrm{mol} / \mathrm{K})$

$T$ : temperature

$T_{\mathrm{c}, i}:$ critical temperature of component $i$

$v$ : molar volume

$x_{i}$ : mole fraction of molecule $i$

$X_{A_{i}}$ : fraction of site $A$ on molecule $i$ that is not bonded to any other components

$z_{i j}$ : coordination number of molecule $i$

$\alpha_{0, i}$ : polarizability of molecule $i$

$\cos \gamma_{i j}$ : angle between the dipole moment of central molecule $i$ and surrounding molecule $j$

$\Gamma_{i}$ : CPA $\Gamma$ parameter for component $i$, related to $a_{0 i}$ and $b_{0 i}$ through $\Gamma_{i}=\left(a_{0 i} / R b_{0 i}\right)$

$\Delta_{A_{i} B_{j}}$ : association strength (equilibrium constant) between site $A$ on molecule $i$ and site $B$ on molecule $j$

$\varepsilon_{\mathrm{r}}:$ static permittivity relative to vacuum

$\varepsilon_{\infty}$ : infinite frequency permittivity relative to vacuum

$\varepsilon_{0}$ : vacuum permittivity $\left(8.85419 \ldots \times 10^{-12} \mathrm{~F} / \mathrm{m}\right)$

$\varepsilon_{A_{i} B_{j}}$ : association energy between site $A$ on molecule $i$ and site $B$ on molecule $j$

$\cos \theta_{i j}$ : hydrogen bond angle between molecule $j$ in the shell around molecule $i$ and the second shell neighbor

$\eta$ : packing factor $\eta=b / 4 \nu$

$\mu_{0, i}$ : dipole moment in vacuum of molecule $i$

$\mu_{0, i}:$ dipole moment vector of central molecule

$\mu_{0, j}^{\prime}$ : dipole moment vector of surrounding molecules

$\nu_{A_{i j} B_{j}}$ : association volume between site $A$ on molecule $i$ and site $B$ on molecule $j$

$\phi_{i}$ : internal $\mathrm{R}-\mathrm{OH}$ bonding angle of molecule $i$

$\rho$ : molar density of fluid

$\mathrm{AAD}$ : absolute average deviation

$\mathrm{RAD}$ : relative average deviation

\section{REFERENCES}

(1) Scaife, B. K. P. Principles of Dielectrics; Oxford University Press: New York, 1998.

(2) Akhadov, Y. Y. Dielectric Properties of Binary Solutions - A Data Handbook; Pergamon Press: New York, 1980.

(3) Haggis, G. H.; Hasted, J. B.; Buchanan, T. S. The Dielectric Properties of Water in Solutions. J. Chem. Phys. 1952, 20, 1452-1466.

(4) Hasted, J. B. In Dielectric and Related Molecular Processes; Davies, M., Ed.; The Chem. Soc.: Burlington House, London, 1972; Vol. 1, Chapter 5, pp 121-162.
(5) Onsager, L. Electric Moments of Molecules in Liquids. J. Am. Chem. Soc. 1936, 58, 1486-1493.

(6) Kirkwood, J. G. The Dielectric Polarization of Polar Liquids. J. Chem. Phys. 1939, 7, 911-920.

(7) Frohlich, H. Theory of Dielectrics; Oxford University Press: London, 1949.

(8) Oster, G.; Kirkwood, J. G. The Influence of Hindered Molecular Rotation on the Dielectric Constants of Water, Alcohols, and Other Polar Liquids. J. Chem. Phys. 1943, 11, 175-178.

(9) Gregory, A. P.; Clarke, R. N. Traceable Measurements of the Static Permittivity of Dielectric Reference Liquids over the Temperature Range 5-50 C. Meas. Sci. Technol. 2005, 16, 1506-1516.

(10) Maribo-Mogensen, B.; Kontogeorgis, G. M.; Thomsen, K. Comparison of the Debye-Hückel and the Mean Spherical Approximation Theories for Electrolyte Solutions. Ind. Eng. Chem. Res. 2012, 51, 5353-5363.

(11) Debye, P.; Hückel, E. Zur Theorie der Elektrolyte. Phys. Z. 1923, 24, 185-206.

(12) Born, M. Volumen und Hydratationswärme der Ionen. Z. Phys. A 1920, 1, 45-48.

(13) Walther, J. V.; Schott, J. The Dielectric Constant Approach to Speciation and Ion Pairing at High Temperature and Pressure. Nature 1988, 332, 635-638.

(14) Djamali, E.; Kan, A. T.; Tomson, M. B. A Priori Prediction of the Thermodynamic Properties of Electrolytes in Mixed AqueousOrganic Solvents to Extreme Temperatures. J. Phys. Chem. B 2012, 116, 9033-9042.

(15) Nelson, S. O. Dielectric Spectroscopy in Agriculture. J. NonCryst. Solids 2005, 351, 2940-2944.

(16) Spies, B. R. Electrical and Electromagnetic Borehole Measurements: A Review. Surv. Geophys. 1996, 17, 517-556.

(17) Buckingham, A. D. A Theory of the Dielectric Polarization of Polar Substances. Proc. R. Soc. London, Ser. A 1956, 238, 235-244.

(18) Suresh, S. J.; Naik, V. M. Hydrogen Bond Thermodynamic Properties of Water from Dielectric Constant Data. J. Chem. Phys. 2000, 113, 9727.

(19) Suresh, S. J.; Naik, V. M. Theory of Dielectric Constant of Aqueous Solutions. J. Chem. Phys. 2002, 116, 4212.

(20) Suresh, S. J. Detailed Molecular Model for Dielectric Constant of Multicomponent, Associating Liquids. J. Phys. Chem. B 2004, 108, $715-720$.

(21) Kontogeorgis, G. M.; Voutsas, E. C.; Yakoumis, I. V.; Tassios, D. P. An Equation of State for Associating Fluids. Ind. Eng. Chem. Res. 1996, 35, 4310-4318.

(22) Kontogeorgis, G. M.; Folas, G. Thermodynamic Models for Industrial Applications; Wiley: Chichester, U.K., 2010.

(23) Von Solms, N.; Michelsen, M. L.; Pereira Passos, C.; Derawi, S. O.; Kontogeorgis, G. M. Investigating Models for Associating Fluids Using Spectroscopy. Ind. Eng. Chem. Res. 2006, 45, 5368-5374.

(24) Soave, G. Equilibrium Constants from a Modified RedlichKwong Equation of State. Chem. Eng. Sci. 1972, 27, 1197-1203.

(25) Wertheim, M. S. Fluids with Highly Directional Attractive Forces. I. Statistical Thermodynamics. J. Stat. Phys. 1984, 35, 19-34.

(26) Chapman, W. G.; Gubbins, K. E.; Jackson, G.; Radosz, M. New Reference Equation of State for Associating Liquids. Ind. Eng. Chem. Res. 1990, 29, 1709-1721.

(27) Haynes, W. M. CRC Handbook of Chemistry and Physics, 93rd ed.; http://www.hbcpnetbase.com (accessed Aug 1, 2012).

(28) Design Institute for Physical Properties (DIPPR) Project 801 Database, 2012 ed. http://www.aiche.org/dippr.

(29) The Landolt-Börnstein Database, http://www.springermaterials. com (accessed Aug 1, 2012).

(30) Foca, G.; Manfredini, M.; Manzini, D.; Marchetti, A.; Pigani, L.; Sighinolfi, S.; Tassi, L.; Ulrici, A. Dielectric Properties in Ternary Mixtures of Ethane-1, 2-diol+ 1, 2-Dimethoxyethane+ Water. Int. J. Thermophys. 2004, 25, 839-855.

(31) Kaatze, U. J. Solution Chem. 1997, 26, 1049-1112.

(32) Yang, J.-Z.; Lu, D.-Z.; Deng, M.; Lui, J.; Li, H.-C.; Hou, C.-J. Dielectric Properties of Urea-Water Mixed Solvents and its Effect on 
the Second Dissociation Process of Carbonic Acid. Z. Phys. Chem. 1998, 205, 199-209.

(33) Hamelin, J.; Mehl, J. B.; Moldover, M. R. The Static Dielectric Constant of Liquid Water Between 274 and $418 \mathrm{~K}$ Near the Saturated Vapor Pressure. Int. J. Thermophys. 1998, 19, 1359-1380.

(34) Fernandez, D. P.; Goodwin, A. R. H.; Levelt Sengers, J. M. H. Measurements of the Relative Permittivity of Liquid Water at Frequencies in the Range of 0.1 to $10 \mathrm{kHz}$ and at Temperatures between 273.1 and $373.2 \mathrm{~K}$ at Ambient Pressure. Int. J. Thermophys. 1995, 16, 929-955.

(35) Lukashov, J. M. Izv. Vyssh. Uchebn. Zaved., Energ. 1976, 20, 89 (adopted from ref 29).

(36) Nicolas, M.; Malineau, R. M. Reich, The Eyring Significant Structure Theory Applied to Methanol-Tetrahydrofuran Mixtures. Phys. Chem. Liq. 1980, 10, 11-22.

(37) Travers, F.; Douzou, P. Dielectric Constants of Alcoholic-Water Mixtures at Low Temperature. J. Phys. Chem. 1970, 74, 2243-2244.

(38) Davidson, D. W. The Dielectric Properties of Methanol and Methanol-d. Can. J. Chem. 1957, 35, 458-473.

(39) Denney, D. J. Dielectric Properties of Some Liquid Alkyl Halides. J. Chem. Phys. 1957, 27, 259-265.

(40) Barbenza, G. H. Dielectric Dispersion in Pure Methyl Alcohol as a Function of Temperature. J. Chim. Phys. Phys.-Chim. Biol. 1968, 65, 906.

(41) Dannhauser, W.; Bahe, L. W. Dielectric Constant of Hydrogen Bonded Liquids. III. Superheated Alcohols. J. Chem. Phys. 1964, 40, 3058-3067.

(42) Corradini, F.; Marchetti, A.; Preti, C.; Tagliazucchi, M.; Tassi, L. Associating Behavior of Mixed Liquids: Dielectric Properties of the Ethane-1, 2-Diol+ 1, 4-Dioxan Solvent System From-10 to $+80^{\circ} \mathrm{C}$. Aust. J. Chem. 1995, 48, 1541-1548.

(43) Krasnoperova, A. P.; Juchno, G. D.; Lebedinez, T. I. Visn. Khark. Univ. 1982, 236, 90 (adopted from ref 29).

(44) Kundu, C. H.; Chattopadhyay, P. K.; Jana, B.; Das, A. N. Thermodynamics of Self-ionization of Ethylene and Propylene Glycols. J. Chem. Eng. Data 1970, 15, 209-213.

(45) Levin, V. V.; Podlovchenko, T. L. Dispersion of the Dielectric Permittivity of ethylene Glycol. Zh. Strukt. Khim. 1970, 11, 766.

(46) Komandin, A. V.; Shimit, B. D. Dielectric Properties of Polyatomic Alcohols in the Liquid State. Zh. Fiz. Khim. 1963, 37, 510.

(47) Koizumi, N.; Hanai, T. Dielectric Properties of Lowermembered Polyethylene Glycols at Low Frequencies. J. Phys. Chem. 1956, 60, 1496-1500.

(48) Akerlöf, G. Dielectric Constants of Some Organic Solvent-water Mixtures at Various Temperatures. J. Am. Chem. Soc. 1932, 54, 41254139.

(49) Smith, R. L., Jr.; Lee, S. B.; Komori, H.; Arai, K. Relative Permittivity and Dielectric Relaxation in Aqueous Alcohol Solutions. Fluid Phase Equilib. 1998, 144, 315-322.

(50) Teutenberg, T.; Wiese, S.; Wagner, P.; Gmehling, J. HighTemperature Liquid Chromatography. Part III: Determination of the Static Permittivities of Pure Solvents and Binary Solvent MixturesImplications for Liquid Chromatographic Separations. J. Chromatogr. 2009, 1216, 8480-8487.

(51) Corradini, F.; Marcheselli, L.; Marchetti, A.; Tagliazucchi, M.; Tassi, L.; Tosi, G. A Non-Linear Correlation Model for the Relative Permittivity of Ternary Amphiprotic (Solvent) Mixtures. Aust. J. Chem. 1993, 46, 1545-1555.

(52) Travers, F.; Douzou, P. Dielectric Constant of Mixed Solvents Used for a Low Temperature Biochemistry. Biochimie 1974, 56, 509514.

(53) Tsierkezos, N. G.; Molinou, I. E. Transport Properties of 2: 2 Symmetrical Electrolytes in (Water+ Ethylene Glycol) Binary Mixtures at $\mathrm{T}=293.15 \mathrm{~K}$. J. Chem. Thermodyn. 2006, 38, 1422-1431.

(54) Douheret, G.; Pal, A. Dielectric Constants and Densities of Aqueous Mixtures of 2-Alkoxyethanols at 25. Degree. C. J. Chem. Eng. Data 1988, 33, 40-43.

(55) George, J.; Sastry, N. V. Partial Excess Molar Volumes, Partial Excess Isentropic Compressibilities and Relative Permittivities of
Water + Ethane-1,2-diol Derivative and Water + 1,2-Dimethoxyethane at Different Temperatures. Fluid Phase Equilib. 2004, 216, 307-321.

(56) Kuila, D. K.; Lahiri, S. C. Comparison of the Macroscopic Molecular Properties in Understanding the Structural Aspects of Mixed Aquo-Organic Binary Mixtures. Z. Phys. Chem. 2004, 218, 803-828.

(57) Yurquina, A.; Manzur, M. E.; Brito, P.; Manzo, R.; Molina, M. A. A. Solubility and Dielectric Properties of Benzoic Acid in a Binary Solvent: Water-Ethylene Glycol. J. Mol. Liq. 2003, 108, 119-133.

(58) Sengwa, R. J.; Madhvi, S. S.; Sharma, S. Characterization of Heterogeneous Interaction Behavior in Ternary Mixtures by a Dielectric Analysis: Equi-Molar H-Bonded Binary Polar Mixtures in Aqueous Solutions. J. Solution Chem. 2006, 35, 1037-1055.

(59) Uosaki, Y.; Kitaura, S.; Moriyoshi, T. Static Relative Permittivities of Water + Ethane-1,2-diol and Water + Propane1,2,3-triol under Pressures up to $300 \mathrm{MPa}$ at $298.15 \mathrm{~K}$. J. Chem. Eng. Data 2006, 51, 423-429.

(60) Travers, F.; Douzou, P.; Pederson, T.; Gunsalus, I. C. Ternary Solvents to Investigate Proteins at Sub-Zero Temperatures. Biochemie 1975, 57, 43-48. 